\title{
Biometric Identification Based on Iris Recognition
}

\author{
Hanaa Fathi Mahmood \\ Department of Computer Science / College of Education \\ University of Mosul
}

Received

$07 / 11 / 2010$
Accepted

02 / 03 / 2011

الملخص

يقدم البحث خوارزمية جديدة لتصنيف صور قزحية العين باستخدام البعد الكسري (خوارزمية

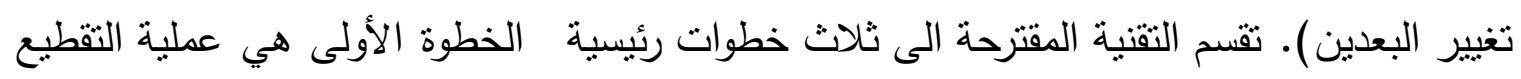

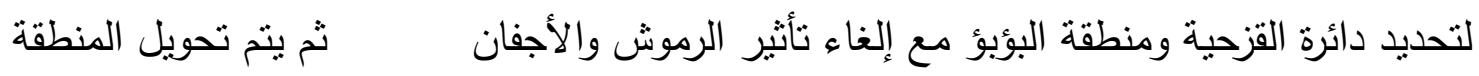

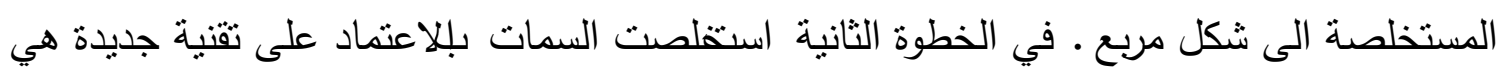
خوارزمية تغيير البعدين لحساب البعد الكسري للقزحية ـوأخيرا استخدمت تقنية Normalized Correlation تم تطبيق الخوارزمية المقترحة في هذا البحث على 990 صور ذات تدرج رمادي من قاعدة بيانات MMU(Multimedia University) التمييز

نتائج الخوارزمية أثنتت ان طريقة تغيير البعدين لحساب البعد الكسري هي طريقة جيدة جدا

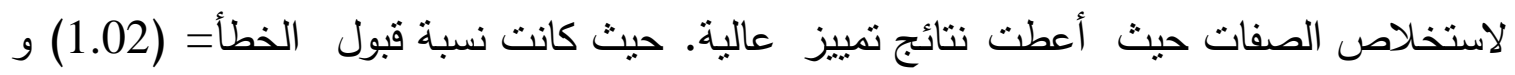
نسبة رفض الخطأ= (0.52) ونسبة تمييز عالية بنسبة (98.45\%).

\section{Abstract}

This paper proposes a novel algorithm for the automatic classification of iris images using a 2_D (two Dimension) Variation to estimate the fractal dimensions of the iris.

The new technique divided in to three main step. In the first step the segmentation process in iris recognition is used to localize the circular iris and pupil regions, excluding eyelids and eyelashes. The extracted iris region is normalized into a squares block with constant dimensions. 
In the second step, the feature extraction techniques are improved and implemented. A new feature extraction technique based on a 2_D Variation to estimate the fractal dimensions is used. Finally The Normalized Correlation is used to classify the iris features. The techniques performed with perfect segmentation on a set of 995 iris images of greyscale eye images from MMU database. The as False Accept Rate $(F A R)$ and False Reject Rate $(F R R)$ and $(R R)$ recognition rate are calculated for this technique. The results of the algorithm proved that. The a 2D (two Dimension) Variation to estimate the fractal dimensions method it is a good feature extraction technique. It gives $F A R=(\mathbf{1 . 0 2})$ and $F R R=(\mathbf{0 . 5 2})$ and a high recognition rate is $\mathbf{9 8 . 4 5} \%$..

\section{Introduction}

Biometrics makes use of certain physiological and behavioral characteristics of a person for identification or verification. These characteristics are called biometric modalities or traits. The physical characteristics like fingerprints, hand geometry, iris, retina, face, hand vein, facial thermo grams, palm print and behavioral characteristics like signature, voiceprint, gait, keystroke dynamics, etc. are examples of biometric modalities [10][12]. Using biometrics for identifying and authenticating human beings offers some unique advantages. Only biometric authentication bases an identification on an intrinsic part of a human being. Tokens, such as smart cards, magnetic stripe cards, physical keys, and so forth, can be lost, stolen, duplicated, or left at home. Passwords can be forgotten, shared, or observed [20][17].

Automated person authentication systems based on iris recognition are reputed to be the most reliable among all biometric methods because of the high level of stability and distinctiveness of the iris patterns [4]. However, the potential requirement of obtaining high accuracy is that users supply iris images of good quality. Current iris recognition systems require only frontal view images of good quality and remove poor quality images by evaluating qualities of images [5] [14] [19].

\section{Related Work}

Using iris patterns as an approach to personal identification and verification goes back to the late 19th century [8], [21], but most work on iris recognition is done in the last decade. Existing methods for iris recognition mainly concentrate on iris representation and matching which is also one of the focuses of this paper. 
Prototype systems had been proposed earlier, John Daugman, implemented a working automated iris recognition system [9][2]. The Daugman system is patented [3]. Daugman's method, based on phase analysis, encodes the iris texture pattern into a 256 byte iris code by using some two dimensional Gabor filters, and taking the Hamming distance [3,4] to match the iris code. Wildes [19] matches images using Laplacian pyramid multi-resolution algorithms and a Fisher classifier. The Daugman system is the most successful and most well know have been developed.

Ma et al. [13] construct a bank of spatial filters whose kernels are suitable for use in iris recognition. They have also developed a preliminary Gaussian-Hermite moments-based method which uses local intensity variations of the iris [11]. They recently proposed an improved method based on characterizing key local variations [9]. Yu et al.[23] use boxcounting method to estimate the fractal dimensions of the iris, then classify iris images into four categories in accordance with the fractal dimensions.

\section{Iris Recognition System}

Generally iris recognition system is composed of many stages as shown in Figure(1). Firstly, an eye image is captured by the system and preprocessed. Secondly, the image is localized to determine the iris boundaries. Thirdly, the iris boundary coordinates are converted to the stretched polar coordinates to normalize the scale and illumination of the iris in the image. Fourthly, features representing the iris patterns are extracted based on texture analysis. Finally, a human is identified by comparing his iris with the iris database [18].

\subsection{Iris Database}

The iris classification algorithm which is proposed in this paper was trained and tested on MMU2 iris database which consists of 995 iris images. The iris images are collected using Panasonic BM-ET100US Authenticam and its operating range is even farther with a distance of $47-53 \mathrm{~cm}$ away from the user. These iris images are contributed by 100 volunteers with different age and nationality. They come from Asia, Middle East, Africa and Europe. Each of them contributes 5 iris images for each eye. There are 5 left eye iris images which are excluded from the database due to cataract disease. Due to some privacy issue. 


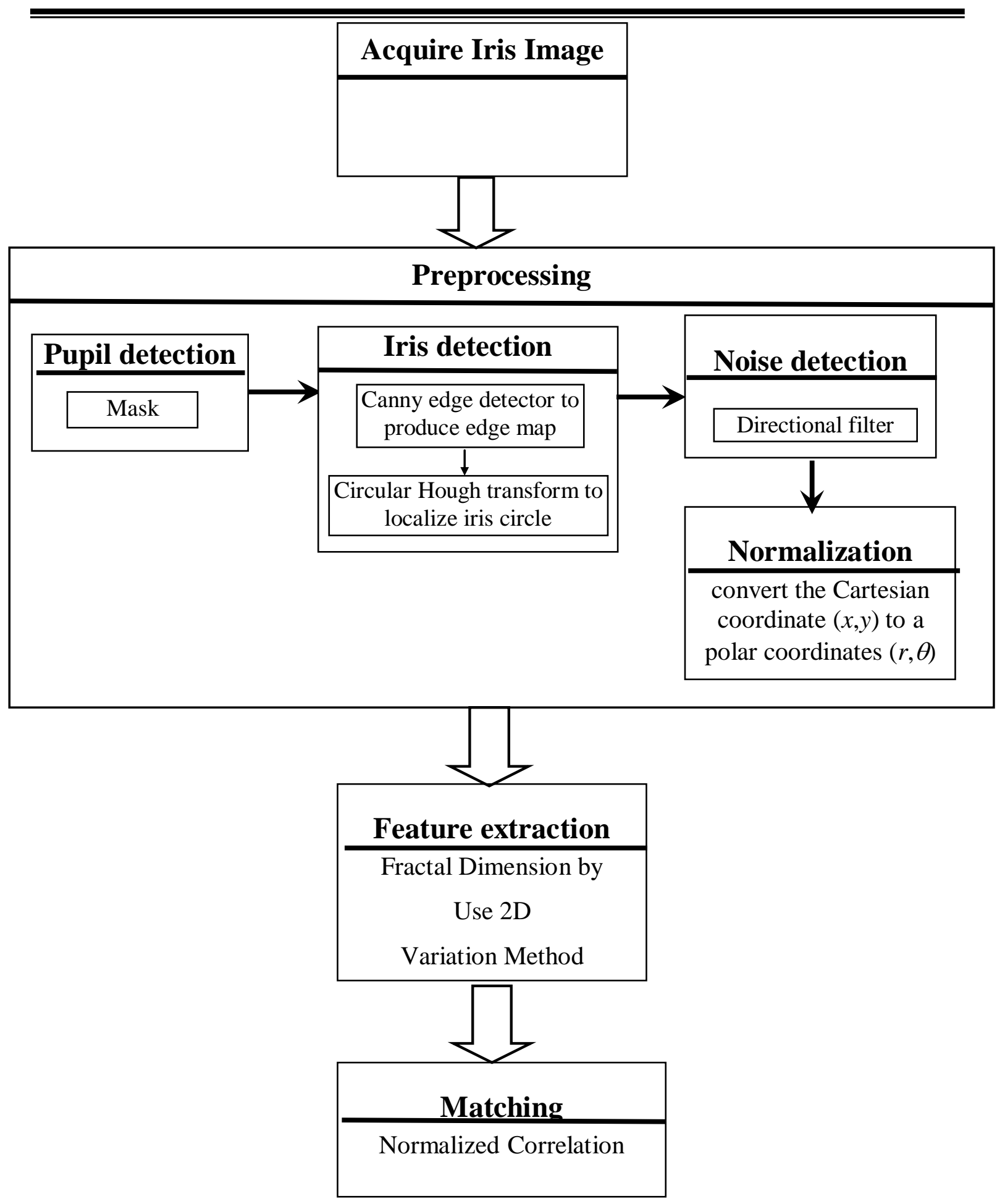

Figure (1): Block diagram of an Iris Recognition System

\subsection{Preprocessing}

The iris region has two main circles, iris circle and pupil circle. The pupil always lies in a dark area in the iris region, an iris also displays a variety of textures which can be broadly classified. 
Pupil Detection by Using $(2 \times 2)$ Mask with ones coefficients, The result of the masked image is threshold, by this the pupil coordinates are detected.

Canny edge detection is used for generate an edge map. Then the circular Hough transform for the iris/sclera boundary is performed. From the above techniques all important parameters are calculated(radius and center coordinates for both pupil and iris circles)[4][5].

After localizing an iris, the inner and outer boundaries are detected. In an eye image, the iris may be partially concealed by the upper eyelid, the lower eyelid, or the eyelash. To exclude these influences, image preprocessing directional filter.

As the size of an iris in a captured image always varies, the detected iris is normalized. The normalization is used to convert the Cartesian coordinate $(x, y)$ to a polar coordinates $(r, \theta)$. Figure $(2 a)$ show normalization technique.

The remapping of the iris region from $(\mathrm{x}, \mathrm{y})$ Cartesian coordinates to the normalized non-concentric polar representation is modeled as

$$
\begin{aligned}
& I(x(r, \theta), y(r, \theta)) \rightarrow I(r, \theta) \\
& \text { with } \\
& x(r, \theta)=(1-r) x_{p}(\theta)+r x_{l}(\theta) \\
& y(r, \theta)=(1-r) y_{p}(\theta)+r y_{l}(\theta)
\end{aligned}
$$

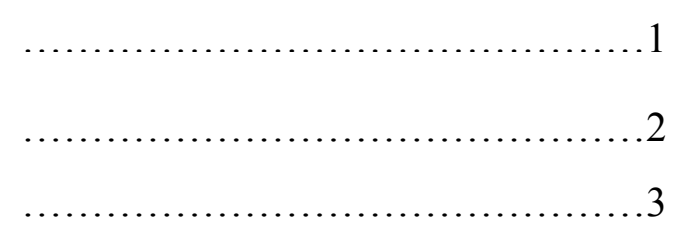

where $I(x, y)$ is the iris region image, $(\mathrm{x}, \mathrm{y})$ are the original Cartesian coordinates, $(r, \theta)$ are the corresponding normalized polar coordinates, and are the coordinates of the pupil and iris boundaries along the $\theta$ direction[23]. Figure (2b) show Cartesian and polar coordinates of an iris.
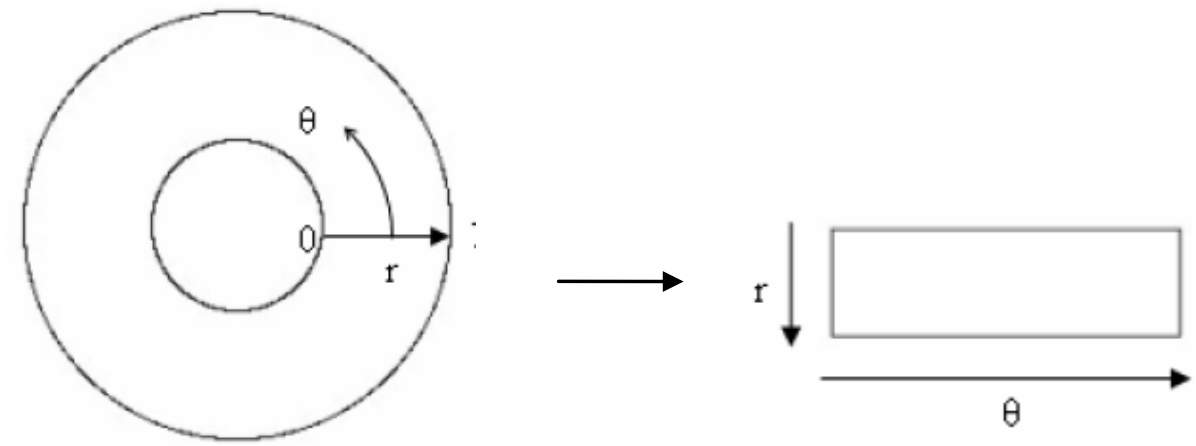

Figure (2a): The normalization technique used to equalize the iris. a): Cartesian.

b): Polar.
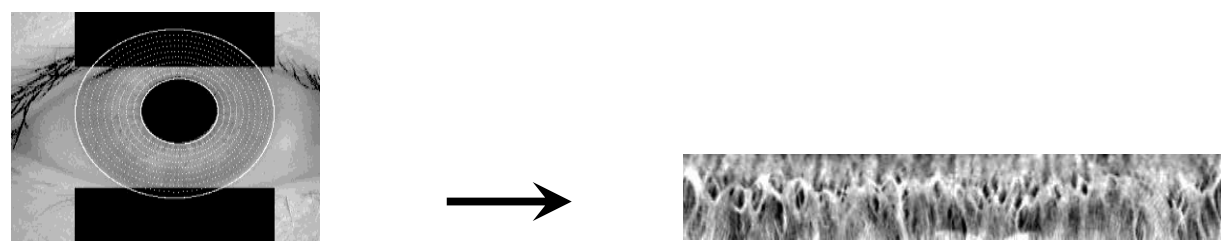

Figure (2b): The Cartesian and polar coordinates of an iris 
Biometric Identification Based on Iris Recognition.

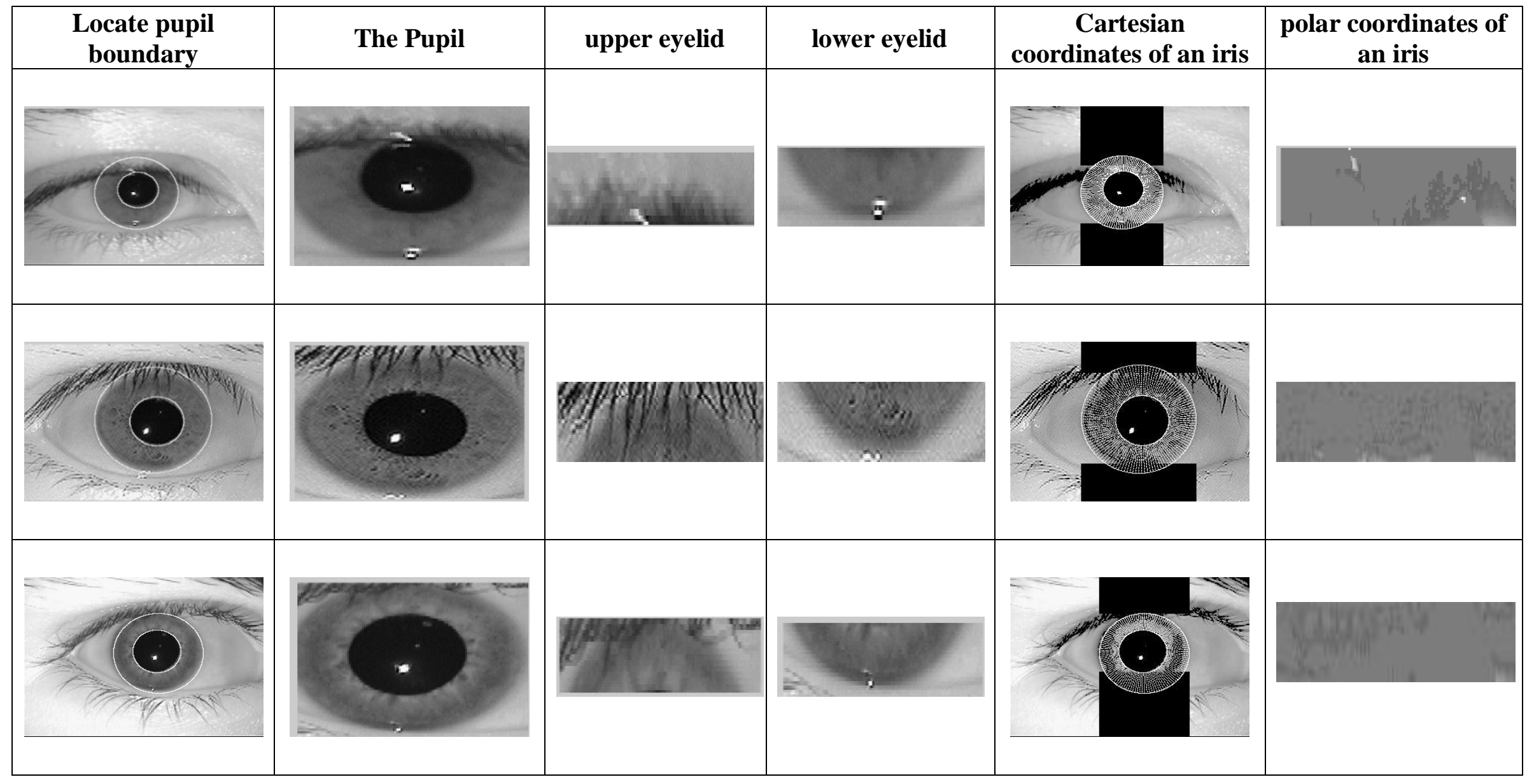

Figure(3): Illustrating the steps involved in the generation of a fixed rectangular enhanced normalized for different iris image.

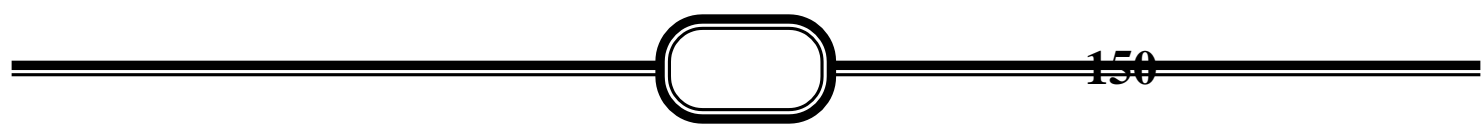




\subsection{Feature Extraction}

An iris image has a unique and complex structure made up of numerous minute interlacing characteristics such as freckles, coronas, furrows, stripes, and crypts. Nonetheless, an iris also displays a variety of textures which can be broadly classified. These textures can be represented numerically, as a calculation of the fractal dimension. The calculation of the fractal dimension begins with preprocessing the original image to localize and normalize the iris[23].

\subsubsection{Fractal Dimension}

In order to understand the fractal dimension exactly in the field of images processing, it is necessary to describe the topological dimension[16][8]. The topological dimension will be the same for a straight line or a curve, whereas the topological dimension for the bi-dimension (dual dimension) shapes or surface will be two like a square, rectangle and the topological dimension for the tri-shapes like the cube and the cylinder will be three. It will be difficult to decide the topological dimension for the landscape (natural shape) like mountains, clouds, trees, sky...etc. As it is difficult to use the traditional geometry in finding the dimension of this sort of shapes the fractal geometry has been used to calculate the fractal dimension of these shape [7][8].

There are three methods used in calculating the fractal dimension of the digital images. these are: The compass method, the Box Counting method, and the 2_D Variation Method [15][8].

\section{- The 2D Variation Method}

The 2D variation method algorithm is an analysis of the pixel environment, and it is one of the best methods used in calculating the fractal dimension of gradating gray image for all scales $\mathbf{r}$ as $\mathbf{r}$ represents the side length of the square surrounding the pixel whose fractal dimension is to be calculated ( $\mathrm{r}$ is calculated by the number of the pixel). To calculate the fractal dimension by using this method, the image centre can be specified by the use of certain scale, and $\boldsymbol{r}$ value is specified then the square gradating value is calculated together with its algorithm. By repeating the process with the change of the scale, it will be possible to approximate $\boldsymbol{r}$ value $(3,5$, $7, \ldots)$; the predilection calculation can be carried out from $\log (1 / r)$ and $\log$ $\mathrm{N}(\mathrm{r})$ such as values as illustrated in the equation and $S$ value will be obtained:

$$
S=\frac{\log N}{\log (1 / r)}
$$


Finally, the fractal dimension is calculated from the following equation $D=3-\frac{S}{2}$

Figure $(\varepsilon)$ illustrates the $2 \mathrm{D}$ variation method by the use of three different dimensions for $\boldsymbol{r}$ [r=5, $\mathrm{r}=7, \mathrm{r}=9]$ [7][8]:

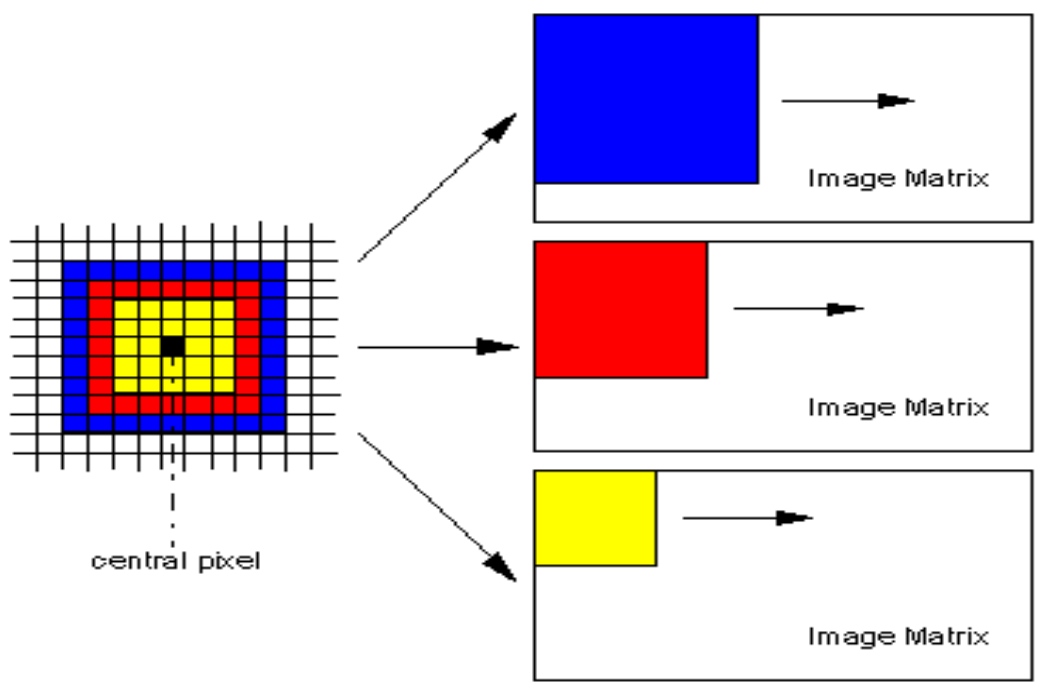

Figure (₹): 2D variation method

\section{Algorithm for Calculating the Fractal Dimension by the Use of 2D Variation Method}

The steps of 2D variation method algorithm are [7][8]:

1- Reading the image and changing its two dimensions into similar dimensions.

2- $\quad$ Finding the image's center pixel.

3- $\quad$ Specifying window $\boldsymbol{w}$ with two dimensions $r$ as $\boldsymbol{r}$ represents the pixel numbers for the window dimension and the window centre is symmetric with the centre of the image.

4- Calculating the number of the pixel which contains the $\mathrm{N}(\mathrm{r})$ texture and which is in the domain of w.

5- $\quad$ Calculating $\log \mathrm{N}(\mathrm{r}), \log (1 / \mathrm{r})$.

6- Increasing the $\mathrm{w}$ window dimension by two pixels and in all directions. The steps from 4 to 6 are repeated until the $\mathrm{w}$ window is covered by the image.

7- $\quad$ Calculating the linear regression $\mathrm{S}$ from $\log \mathrm{N}(\mathrm{r})$ values by depending on the scale values of $\log (1 / \mathrm{r})$. 


\section{Hanaa Fathi Mahmood}

8- Calculating the fractal dimension from the following equation:

$$
D=3-\frac{S}{2}
$$

\section{Classifying an iris using the Normalized Correlation:}

Normalized correlation between the acquired and database representation was used for goodness of match [21]. This is represented by the following equation:

$$
N C=\frac{\sum_{i=1}^{n} \sum_{j=1}^{m}\left(p_{1}[i, j]-\mu_{1}\right)\left(p_{2}[i, j]-\mu_{2}\right)}{n m \sigma_{1} \sigma_{2}}
$$

$\sigma_{1}$ are the mean and standard deviation of $p_{1}$, and $\mu_{2}$ and $\sigma_{2}$ are the mean and standard deviation of $p_{2}$.Normalized correlation is advantageous over standard correlation, since it is able to account for local variations in image intensity that corrupt the standard correlation calculation [21].

\section{Experimental Results}

Tests are carried out to confirm that iris recognition can perform accurately as a biometric for recognition of individuals. Experiments are also conducted in order to confirm the uniqueness of human iris patterns.

To obtain the maximum features in the irises, the iris region is normalized to $256 \times 64$ for radial and angular resolution for the Fractal Dimension implementation, a preprocessed iris image are divided into blocks.. $128 \times 64$ image blocks were obtained from original iris image. This blocks are concatenate to convert image to a square shape $(128 \times 128$ pixels). Then 2D Variation method were used with different windows size $(5,7,9,13,17,19.23)$ to calculate the fractal dimensions of these image blocks.

Errors and recognition rate for the proposed and improved techniques are investigated. Errors are known as False Accept Rate $(F A R)$ and False Reject Rate $(F R R)$ which are given by [22]:

$$
\begin{aligned}
& F A R=\frac{\text { Number of incidents of false accepted }}{\text { Total number of samples }} \times 100 \% \ldots(7) \\
& F R R=\frac{\text { Number of incidents of false rejected }}{\text { Total number of samples }} \times 100 \% \ldots(8)
\end{aligned}
$$

while the recognition rate is given by:

$$
R R \%=100-(F A R+F R R)
$$


Table(1) shows the performance of the new algorithm which proposed in this paper with different window size. The proposed algorithm gives a recognition rate of $\mathbf{9 8 . 4 5} \%$ with FAR of $\mathbf{1 . 0 2 2} \%$ and FRR of $\mathbf{0 . 5 2} \%$.

Table (1): shows the performance with different window size

\begin{tabular}{||c|c|c|c||}
\hline Window size & FAR & FRR & RR \\
\hline 5 & 8.4335 & 0 & 91.5665 \\
\hline 7 & 1.022381 & 0.522321 & 98.455298 \\
\hline 9 & 6.191655 & 1.832571 & 91.975774 \\
\hline 13 & 0 & 3.370787 & 96.629213 \\
\hline 17 & 7.228916 & 0 & 92.771084 \\
\hline 19 & 0.787402 & 1.574803 & 97.637795 \\
\hline 23 & 4.36659 & 1.33782 & 94.29559 \\
\hline
\end{tabular}

\section{Conclusion}

The human iris contains a very unique pattern that can be used as the basis for biometric identification of individuals. this paper presents a novel iris classification algorithm based on 2D (two Dimension) Variation method of fractal dimension. The iris recognition system has revealed a number of interesting conclusions. It can be stated that segmentation is the critical stage of iris recognition, since areas that are wrongly identified as iris regions will corrupt biometric resulting in very poor recognition.

The classification method has been tested and evaluated of 995 iris images of greyscale eye images from MMU database. the best result was obtained using the window size 7 which was $98.45 \%$ accurate. It is a good performance for iris identification. 


\section{References}

1) Byungjun Son, Sung-Hyuk Cha, Yillbyung Lee. 2007, "DLDA-based Iris Recognition from Image Sequences with Various Focus Information", Proceedings of the 7th WSEAS International Conference on Multimedia Systems \& Signal Processing, Hangzhou, China.

2) Daugman John and Downing Cathryn, 1994, "Recognizing Iris Texture By Phase Demodulation", Image Processing for Biometric Measurement, IEE Colloquium on, pp., 2/1-2/8.

3) Daugman John G., 1993, "High Confidence Visual Recognition of Persons by a Test of Statistical Independence", IEEE Transaction on Pattern Analysis and Machine Intelligence, Vol. 15, no. 11, pp. 1148-1161.

4) Daugman, J. G. 2001, Statistical richness of visual phase information, International Journal of Computer Vision, 45(1),, pp. 25-38.

5) Daugman, J. G 1993, High confidence visual recognition of persons by a test of statistical independence, IEEE Trans. on Pattern Analysis and Machine Intelligence, 15, pp. 1148-1161.

6) Deb Sagarmay, 2004, "Multimedia Systems and Content-Based Image Retrieval”, Idea Group Inc. (IGI).

7) Ekhlass Abd Al-Gabar,2004, " Natural Object Recognition For Digital Image", Ms. C. thesis, Department of computer science, College of computers \& mathematical Sciences, university of mosul.

8) Fawziya M. Ram 2006, "Civil Objects Recognition Based on Proposed Hybrid Technique" Ph.D. Thesis Department of computer science, College of computers \& mathematical Sciences, university of mosul

9) Francis Galton, 1989, "Personal Identification and Description", Journal of the Anthropological Institute of Great Britain and Ireland, Vol. 18, pp. 177-191.

10) Jain, A. K., Bolle R. and Pankanti, S. 1999, BIOMETRICS : Personal Identification in Networked Society, Kluwer Academic Publishers.

11) Lisa Kuster, 2003, "An Overview of Biometric Technologies", GSEC Practical Assignment, Version 1.4b Option 1.

12) Nakamura Y.,Sharma C, 2003,"Wireless Data Services: Technologies, Business Models and Global Markets", Cambridge University. 
13) Ma Li, Tan Tieniu, Wang Yunhong, and Zhang Dexin, 2003, "Personal Identification Based on Iris Texture Analysis", IEEE Transactions on Pattern Analysis and Machine Intelligence, Vol. 25, No. 12, pp. 1519-1533.

14) Negin, M. T. Chmielewski, M. Salgnicoff, U. von Seelen, P. Venetainer and G. Zhang, 2000, "An iris biometric system for public and personal use", In IEEE Computer, 33, pp. 70-75

15) Rayan Yousif, 2003," Fractal Dimension Calculation for the General Shapes of the Borders of Geographical Maps and Closed Curves", M.Sc thesis, Department of computer science, College of computers $\&$ mathematical Sciences, university of mosul.

16) Shaima Mudhar Y.Al-Norri, 2004, " Digital Image Compression Based on Wavelet Transform and Fractals", Msc. Thesis, Department of computer science, College of computers \& mathematical Sciences, university of mosul.

17) Saday Taha, Akhan Nurdan, 2003, "Biometric Recognition Systems", IJCI Proceedings of International Conference on Signal Processing, Vol 1, No. 2, pp. 136-139.

18) Sharp Robin, 2005, "User Authentication", Computer Security (C, Informatics and Mathematical Modeling, Technical University of Denmark.

19) Wildes R. P.1997, Iris Recognition : An emerging biometric technology, Proc. of the IEEE, 85(9), pp. 1348-1363.

20) Wikipedia Contributors, "Biometrics Information on Wikipedia.com", Wikipedia, The Free Encyclopedia", Update: March 2006, Cited at: http://en.wikipedia.org/wiki/Biometrics.

21) Wildes Richard P., 1997, "Iris Recognition: An Emerging Biometric Technology", Proceedings of the IEEE, Vol. 85, No.9, pp. 1348-1363.

22) Woodward John, Orlans Nicholas M., Higgins Peter T., 2003, "Biometrics", McGraw-Hill Professional.

23) Yu, L., Zhang, D., Wang, K., Yang, W. 2005, Coarse iris classification using box-counting to estimate fractal dimensions. Pattern Recognition 38, 1791-1798. 